\title{
Jeux européens et innovation institutionnelle. Les logiques de création d'Eurojust (1996-2004)
}

\section{Michel Mangenot}

\section{(2) OpenEdition \\ 1 Journals}

\section{Édition électronique}

URL : http://journals.openedition.org/conflits/2056

DOI : 10.4000/conflits.2056

ISSN : $1777-5345$

Éditeur :

CCLS - Centre d'études sur les conflits lilberté et sécurité, L'Harmattan

\section{Édition imprimée}

Date de publication : 1 juin 2006

Pagination : 43-62

ISBN : 2-296-01073-3

ISSN : 1157-996X

\section{Référence électronique}

Michel Mangenot, « Jeux européens et innovation institutionnelle. Les logiques de création d'Eurojust (1996-2004) », Cultures \& Conflits [En ligne], 62 I printemps 2006, mis en ligne le 19 juillet 2006, consulté le 30 mars 2021. URL : http://journals.openedition.org/conflits/2056 ; DOI : https://doi.org/ $10.4000 /$ conflits.2056

Ce document a été généré automatiquement le 30 mars 2021.

Creative Commons License 


\title{
Jeux européens et innovation institutionnelle. Les logiques de création d'Eurojust (1996-2004)
}

\author{
Michel Mangenot
}

1 Eurojust est le nouvel organe de coopération judiciaire en matière pénale de l'Union européenne. Issu d'une décision du Conseil du 28 février 2002, doté de la personnalité juridique, son siège est, depuis décembre 2002, à La Haye. Son objectif est d'améliorer la coordination entre procureurs et enquêteurs nationaux travaillant sur des dossiers de criminalité transfrontalière grave et de leur prêter son concours en vue de renforcer leur efficacité. Dans cet article, nous nous intéresserons aux conditions et aux logiques d'invention et d'élaboration d'Eurojust au sein de l'espace judiciaire pénal européen. Cette création apparaît comme la dernière étape d'un processus de coopération institutionnelle judiciaire entamé dans le cadre du troisième pilier de l'Union européenne en novembre 1993 avec la mise en œuvre du traité de Maastricht. Ces étapes sont les suivantes : création des magistrats de liaison (1996) ${ }^{1}$, réseau judiciaire européen (1998) ${ }^{2}$ et Eurojust. Ce projet apparaît à ce jour, et pour plusieurs années encore, comme le plus «intégré » en matière de coopération judiciaire pénale. Ajouté au traité CE dès Nice (février 2001), il est inscrit dans le projet de traité constitutionnel signé le 29 octobre 2004 à Rome (article III-273).

2 A la différence du projet du Procureur européen, par exemple, ce projet a abouti. Cette réussite lui vaut de nombreuses revendications de paternité. Ainsi trouvera-t-on aussi bien des revendications française et allemande que belge. Ces multiples revendications s'expliquent par le fait qu'Eurojust n'a été pendant longtemps qu'une appellation, qu'un label. Si le terme a vraisemblablement été inventé au moment de la préparation du Conseil européen de Tampere en 1999, l'idée remonte à l'annonce, en 1991, d'un « FBI européen » par Helmut Kohl et la création d'Europol par le traité de Maastricht en 1992. C'est à cette période que naît l'idée d'une agence européenne qui serait l'équivalent d'Europol dans le domaine judiciaire. Pour reprendre le terme employé par l'un de nos interlocuteurs dans un entretien, Eurojust est «inscrit dans les astres 
depuis Europol ${ }^{3}$ ». Au lendemain même de la mise en œuvre du traité de Maastricht, le 2 novembre 1993, une première initiative a été évoquée comme " origine » d'Eurojust. Le ministre belge de la Justice propose alors une action commune établissant un "Centre pour l'information, l'étude et l'échange dans le domaine de la coopération judiciaire ", dénommé CIREJUD. Le modèle était celui fourni par des structures déjà existantes dans le domaine de l'asile (CIREA) et de l'immigration (CIREFI). Mais cette proposition aboutit en fait, en 1998, à un réseau de points de contact appelé « réseau judiciaire européen » (RJE).

3 Pour se garder d'un récit héroïque porté par de nombreux acteurs inter-rogés ${ }^{4}$ et restituer le plus finement possible cette sociologie de la décision, nous avons choisi une présentation en termes de jeux institutionnels. Ces jeux sont relativement autonomes et connectés, dans le sens où les acteurs et les espaces dans lesquels ils évoluent sont différenciés et qu'ils disposent de règles et d'enjeux propres. Des coups isolés au sein de l'un des jeux provoquent des effets dans les autres. Des coups jouables dans l'un ne le sont pas dans un autre. C'est de l'interaction et de l'unification de ces jeux que provient Eurojust. Si ces cinq jeux ont des temporalités différentes, certains se (re)jouent dans une même séquence décisive (juillet-décembre 2000).

Le premier de ces jeux s'organise autour du « leadership organisationnel », suivant les termes de Philip Selznick ${ }^{5}$, d'un organe communautaire discret - le Secrétariat général du Conseil - et de la socialisation et spécialisation d'un groupe aux ressources intellectuelles élevées et intéressé par le travail «noble » de création et d'ingénierie institutionnelle. Les autres jeux sont déterminés par une forte concurrence interinstitutionnelle avec la Commission inscrite dans une logique autonome et ancienne, ou marqués par un rythme propre (celui de la présidence ou des présidences) ou encore, déterminés par une conjoncture spécifique comme la Conférence intergouvernementale (CIG) qui, seule, permet certains coups.

5 On s'insère ici par ailleurs dans les perspectives développées par H. Jamous, pour lequel les dysfonctionnements ne sont pas cause du changement mais des instruments dont se saisissent des groupes sociaux ${ }^{6}$. Pour lui, le rôle des personnalités est déterminant, tout autant que leur pouvoir charismatique. Ici, les membres du SGC sont des médiateurs ou des marginaux sécants entre champs administratif, judiciaire et universitaire (avec une séparation entre national et européen pour le premier champ). Ils s'entourent ou s'allient avec des prétendants: des fonctionnaires de la justice ou des magistrats fonctionnarisés. Si l'on reprend les catégories de H. Jamous, il s'agit de catégories sociales et professionnelles porteuses de nouvelles valeurs en décalage par rapport à un système (ici judiciaire) traditionnel. Ces nouvelles valeurs seraient celles de l'européanisation et, plus spécifiquement, de l'invention de nouveaux instruments, audelà de la simple coopération interpersonnelle.

6 Ainsi, nous nous situons dans une approche où les individus jouent un rôle parfois déterminant, mais dont le pouvoir personnel s'explique principalement par leur insertion et leur place dans des jeux institutionnels. De ce point de vue, il apparaît que la force des acteurs du Secrétariat général, et d'une certaine façon le charisme qui leur est prêté, réside dans leur présence dans l'ensemble des jeux, à l'exception notable d'un seul : celui de la CIG, en raison justement de l'élévation du niveau de la décision qu'il opère. En tant que producteurs des caractéristiques formelles de l'organisation, ils contribuent ainsi à structurer (autour d'eux) les espaces de négociations et de jeu ${ }^{7}$. Ils déterminent à la fois les « comportements organisationnels ", et apparaissent comme 
les acteurs inchangés des processus d'interactions et de marchandages au sens, respectivement, du deuxième et troisième modèle de la sociologie de la décision d'Allison ${ }^{8}$.

Agenda et cadrage : le jeu du Secrétariat général du Conseil

7 Le premier jeu illustre le rôle d'entrepreneur conquis par le Secrétariat général du Conseil ${ }^{9}$ et sa direction générale Justice et Affaires intérieures (JAI). Le Secrétariat du Conseil dispose en effet depuis 1995 d'une direction générale spécialisée, à la différence de la Commission européenne. Fin 1994, le nouveau Secrétaire général du conseil décide d'élever au rang de Direction générale la petite direction JAI créée en novembre 1993. Alors que le premier responsable n'était pas un spécialiste (représentant permanent adjoint de la Belgique, ancien inspecteur des Finances), on assiste alors à un recrutement de haut niveau et aux compétences judiciaires très élevées. Charles Elsen, chargé de ces questions au ministère luxembourgeois de la Justice depuis trente ans et, à ce titre, fondateur de TREVI ${ }^{10}$, est nommé Directeur général. Julian Schutte, ayant, lui, vingt ans d'expérience similaire au ministère néerlandais de la Justice et ayant été co-rédacteur de la convention Schengen, est alors nommé directeur juridique. Proche des ministres, il a contribué à déterminer la politique judiciaire des Pays-Bas. Le cas le plus emblématique est celui de Gilles de Kerchove, assistant à l'université catholique de Louvain, chef de cabinet du ministre belge de la Justice depuis 1989 - et, à ce titre, associé à la proposition de 1993 de CIREJUD -, qui est nommé en septembre 1995 directeur de la coopération judiciaire et policière. Il était alors en concurrence pour ce poste avec une maître des requêtes au Conseil d'Etat de France. L'équipe se complète enfin en juillet 1996 avec la nomination de Hans Nilsson, juge de Cour d'appel suédois, spécialiste de droit pénal, passé par le Conseil de l'Europe où il était en poste depuis dix ans. Il est recruté comme chef de la division « Coopération judiciaire ». La perspective est fondamentalement différente de celle de la Commission. Adrian Fortescue, diplomate britannique issu de la London School of Economics, détaché à la Commission depuis 1985, chef de cabinet de Lord Cockfield, Commissaire au Marché intérieur (1985-1989), est nommé chef de la Task Force JAI au Secrétariat général de la Commission en 1994, puis premier directeur général en $1999^{11}$. La nouvelle chef de l'unité « Coopération judiciaire », Gisèle Vernimmen, est elle, issue de la DG du Marché intérieur. Ces deux hauts fonctionnaires ne disposent pas de compétences judiciaires ${ }^{12}$. C'est dans cette configuration institutionnelle qu'interviennent les événements de l'automne 1996. Alors que la présidence irlandaise avait choisi comme thème prioritaire la lutte contre la drogue, Véronica Guérin, célèbre journaliste, est assassinée en Irlande par des trafiquants de stupéfiants. Par ailleurs, dans le cadre de l'affaire Dutroux, la marche blanche à Bruxelles a lieu, rassemblant 300000 personnes. «Cela a causé un tollé en Irlande qui a décidé de consacrer une partie de son Conseil européen de Dublin, Dublin II, à la lutte contre la criminalité organisée. Et la présidence avait décidé de faire une réunion du Comité $\mathrm{K} 4$ [un comité de coordination à haut niveau] ${ }^{13}$ " se souvient Hans Nilsson.

9 Dans la perspective de cette réunion du 15 octobre 1996, l'équipe du Secrétariat général se saisit de cette mise sur agenda de la « criminalité organisée " pour, selon les propos figurant dans l'un de nos entretiens, "maximiser l'événement». Elle diffuse parmi certaines délégations un papier qui propose la mise en place d'un système d'évaluation mutuelle en matière d'entraide judiciaire mais, surtout, la création d'une Unité de coopération judiciaire (JCU en anglais, "Judicial Cooperation Unit»). Il s'agit d'une 
fenêtre d'opportunité pour le Secrétariat général. Ce document est alors longuement débattu par le Comité K4, mais rejeté par quelques délégations. Cette idée n'apparaît donc plus au Conseil européen de Dublin de décembre 1996, qui décide cependant de créer un groupe à haut niveau, chargé de rédiger un programme d'action de lutte contre la criminalité organisée. Il deviendra, sous présidence luxembourgeoise, le "Groupe multidisciplinaire sur la criminalité organisée ", le GMD. Cela aboutit en réalité à consolider et à autonomiser définitivement la lutte contre la criminalité organisée dans la coopération policière ${ }^{14}$.

C'est en effet en relation avec des acteurs plus spécifiquement judiciaires qu'émergera Eurojust, en particulier au sein d'une nouvelle enceinte : le groupe de travail du Conseil "Coopération judiciaire pénale", créé par la présidence irlandaise dans ce même contexte de la préparation du Conseil européen de Dublin ${ }^{15}$. C'est dans cette perspective que de nouveaux acteurs sont arrivés à Bruxelles dans les Représentations permanentes: Emmanuel Barbe, qui inaugure le poste de conseiller Justice en 1997 pour la France, Lorenzo Salazar pour l'Italie; dans un autre cadre, Dan Eliason pour la Suède, et Daniel Flore pour la Belgique, tous deux en tant que délégués de leur Etat au Comité K4. Ce dernier, directement en poste au ministère de la Justice de son pays du fait même de sa localisation, suit les questions européennes depuis plus longtemps (1985).

11 La mise en œuvre du traité d'Amsterdam en mai 1999 est concomitante de la présidence finlandaise. Or, celle-ci décide d'organiser le premier Conseil européen entièrement autour des questions de «Justice et d'Affaires intérieures». Dans ce cadre, le Secrétariat général du Conseil bénéficie d'une grande confiance de la part de la présidence finlandaise. Au sein du SG, le duo formé par de Kerchove et Nilsson réunit une petite équipe pour tester leurs idées sur cette Unité de coopération judiciaire, déjà proposée fin 1996 à la présidence irlandaise. Le terme d'» Eurojust ", en référence à Europol, apparaît alors dans ces discussions informelles. Se noue ici un réseau de relations professionnelles, personnelles et même d'amitié. En plus d'investissements universitaires ${ }^{16}$, ce réseau est engagé dans une stratégie éditoriale au sein de la collection «Etudes européennes" des éditions de l'université de Bruxelles. Dans ce système relationnel, les deux parties sont mues par des intérêts réciproques. Pour de Kerchove et Nilsson, il s'agit de développer le rôle du Secrétariat général, de s'appuyer sur cette expertise nationale et de savoir en quelque sorte avant les négociations ce qui est susceptible d'aboutir à un accord. Les conseillers Justice, qui ont occupé des postes de magistrats sur des dossiers très techniques, sont intéressés à faire de la coopération institutionnelle.

12 Il s'agit maintenant de faire avaliser ce projet par la présidence finlandaise, qui reste maîtresse de l'initiative et du contrôle de l'agenda. Le rôle très spécifique du Secrétariat général dans la préparation des Conseils européens est poussé à son maximum. Le travail consiste à rédiger les conclusions de la présidence dans les mois qui précèdent. Ici, la conjoncture est encore plus spécifique puisqu'un Conseil européen ne rassemble pas les ministres de la Justice. Ainsi, une réunion informelle des ministres de la Justice et de l'Intérieur a lieu à Turku, en Finlande, en septembre 1999, un mois avant le sommet de Tampere. Il s'agit d'une consultation des ministres «sectoriels» pour préparer le Conseil européen. Cette réunion informelle aboutit à la première officialisation politique d'Eurojust. L'équipe de la DG JAI est ici secondée par un ancien membre du ministère allemand de la Justice et membre du cabinet du Secrétaire 
général du Conseil, également allemand, $\mathrm{M}$. Trumpf ${ }^{17}$. Arrêtons-nous un instant sur la mise en récit de cet événement par un membre du Secrétariat général du Conseil :

« Nous avions préparé, pour le débat de la première matinée, une série de questions destinées à faire émerger un consensus sur la création d'Eurojust. Nous fûmes très étonnés de constater qu'aucun ministre n'en évoqua même l'idée. Comme nous savions que la ministre allemande de la Justice était acquise à cette idée - elle en avait été persuadée par un haut magistrat allemand ${ }^{18}$ \# - nous sommes allés lui parler avant le déjeuner pour lui exprimer notre étonnement. Nous avons également attiré l'attention du conseiller de la ministre française de la Justice, tout acquis lui aussi à l'idée. Lors de la reprise des travaux, et bien que ceux-ci étaient consacrés aux questions d'immigration et d'asile, la ministre allemande de la Justice a repris la parole et insisté sur l'importance de la création d'Eurojust. Elisabeth Guigou lui a apporté son soutien et, à la suite de son intervention, douze autres ministres de la Justice ont indiqué qu'ils trouvaient l'idée intéressante. Ceci a contribué à convaincre la présidence finlandaise d'insérer le principe de la création d'Eurojust dans les conclusions du Conseil européen de Tampere. La Finlande n'en voyait pas en effet la nécessité, son système judiciaire ne prévoyant pas de rôle spécifique pour le parquet pendant la phase d'enquête et d'instruction ${ }^{19}$ ".

On voit bien ici le jeu complexe dans lequel agissent les agents du Secrétariat général. Leur position leur permet de se faire prévaloir, selon les circonstances, de conseiller de la présidence ou des délégations nationales d'autant plus facilement qu'ils n'ont institutionnellement aucun rôle bien défini (une présidence peut se passer, si elle le souhaite, de toute aide, autre que purement logistique, du SGC) et aucune position officielle à défendre (contrairement à la Commission). Ils peuvent ainsi faire naître une demande.

C'est aussi dû à la souplesse de l'organisation administrative du Secrétariat qui n'a pas, selon les personnes interrogées, les mêmes pesanteurs que la Commission. Comme l'explique un membre du SGC, ici «on n'a pas besoin de consulter de multiples personnes, on le propose à la présidence, elle le fait sien ou non, elle le dépose et c'est fini : ça prend deux jours. A la Commission, ça prend six mois parce qu'il y a toute la machine, la hiérarchie, le service juridique, le cabinet, le collège ». Une organisation faiblement codifiée comme le Secrétariat peut agir plus efficacement dans une configuration institutionnelle incertaine. Cela ne veut pas dire pour autant qu'il y ait absence de conflits : on a ainsi assisté au SGC à des divergences internes sur Eurojust entre la DG JAI et le service juridique, en l'occurrence Julian Schutte, directeur ayant côtoyé Nilsson au Conseil de l'Europe à Strasbourg lorsqu'il représentait les Pays-Bas.

C'est bien, pour la mise sur l'agenda d'Eurojust, à une imposition à laquelle on a assisté. Cette imposition est loin d'être évidente pour tous les sujets, car une autre proposition du Secrétariat général du Conseil - la création d'une grande école de la magistrature européenne - n'est pas reprise, faute de consensus minimum de la présidence ou d'une majorité d'Etats membres. Ici, c'est aussi l'appui franco-allemand (recherché d'ailleurs par le SGC) qui permet l'inscription d'Eurojust dans les conclusions de la présidence de Tampere. Dans ce premier jeu, le Secrétariat général semble avoir été au centre de la configuration institutionnelle et intellectuelle. C'est des interactions entre différentes présidences que surgit la proposition formelle de création.

Le temps des présidences 
17 Le deuxième jeu est celui de la présidence (de l'UE), plus précisément de quatre présidences - Portugal, France, Suède, Belgique - coordonnées pour la proposition formelle d'Eurojust, et même cinq, avec l'initiative concurrente de l'Allemagne. Dans ce jeu, le Secrétariat général ne peut plus être le pivot central des réflexions. C'est le rôle des présidences qui est ici déterminant, dans la mesure où chacune souhaite imprimer ses propres priorités et faire avancer ses projets.

18 La présidence portugaise souhaite mettre sur la table une proposition qui, d'emblée, rassemblerait un certain consensus. Elle convoque une réunion à sa Représentation permanente et convie les trois présidences suivantes : France, Suède et Belgique. Tout en souhaitant s'appuyer sur d'autres partenaires pour s'assurer d'un bon accueil du projet, la présidence portugaise est aussi tenue par l'objectif de Tampere. Le cadrage imposé par les chefs d'Etat et de gouvernement à Tampere (en fait par le Secrétariat général) est extrêmement précis au niveau formel, puisqu'il indique que le conseil devra adopter l'instrument légal nécessaire avant la fin 2001, c'est-à-dire sous la présidence belge. Les contacts informels développés avant Tampere à l'initiative du SGC se trouvent formalisés par ce cadrage qui détermine rapidement une prise en charge du dossier par les présidences jusqu'au délai imparti, dans l'ordre : Portugal, France, Suède et Belgique. C'est dans ce sens qu'il faut comprendre la phrase de de Kerchove : "Nous avons réuni ici trois ou quatre délégations d'Etats membres pour rédiger ensemble le projet que déposeraient leurs Etats respectifs, à savoir la France, la Suède, le Portugal et la Belgique ». L'influence du SGC se retrouve ainsi au travers d'effets d'anticipation sur les calendriers européens dans la gestion et la prévision des agendas, étant donné les délais de plus en plus longs de prise de décision à Bruxelles.

19 Mais l'espace institutionnel est bien celui de la présidence et, en particulier ici, des quatre présidences. Le coup de Turku ne peut être joué qu'une fois. Il revient bien à la présidence portugaise de mener et de coordonner les travaux préparatoires à une initiative conjointe. C'est une membre du ministère de la Justice à Lisbonne, et non de la Représentation permanente à Bruxelles, Madame Theresa Alvez Martins, directrice des relations internationales, qui est la coordinatrice. La présidence choisit une formule ouverte de mise à la discussion d'une série d'options. Dès le 4 février 2000 , elle soumet au Comité de l'article $36^{20}$ des scénarios concernant uniquement la détermination des compétences ratione materiae d'Eurojust et la définition de ses pouvoirs. C'est sur cette base qu'est proposé un questionnaire aux ministres de la Justice les 3 et 4 mars 2000, lors d'une réunion informelle à Lisbonne.

Il faut attendre la présidence française début juillet pour qu'un texte mûr soit déposé au Conseil le 20 juillet $2000^{21}$. Publié au Journal officiel le 24 août, il est signé des quatre présidences ${ }^{22}$. Cette initiative prévoit l'institution d'une Unité de coordination judiciaire «Eurojust », composée d'un membre national par Etat membre, ayant la qualité de procureur, de magistrat ou d'officier de police, pour des formes de criminalité affectant deux ou plusieurs Etats membres et nécessitant une action coordonnée des autorités judiciaires. Sa compétence matérielle est précisée : les types de criminalité et d'infractions couverts par la convention Europol ; la traite des êtres humains ; les actes de terrorisme; la protection de l'euro; la protection des intérêts financiers de la Communauté ; le blanchiment d'argent; la criminalité informatique et d'autres formes de criminalité en relation avec les infractions susmentionnées. Elle prévoit qu'Eurojust pourra demander (de façon non contraignante) à un Etat membre d'entreprendre une enquête ou des poursuites dans un cas précis, ou à plusieurs Etats 
membres de coordonner leurs activités d'enquête et de poursuite. Si l'Etat membre refuse d'entreprendre l'enquête, il devra motiver sa décision. Enfin, ce texte précise qu'Eurojust disposera de la personnalité juridique et sera dirigé par un président, assisté de deux vice-présidents, tous trois choisis par le Conseil parmi les membres nationaux. Mais ce texte des quatre présidences, préparé depuis des mois, est courtcircuité par une proposition allemande déposée un mois auparavant, sans concertation avec les quatre présidences. Le 19 juin en effet, dans l'urgence, l'Allemagne officialise une proposition propre sur Eurojust (publiée le 19 juillet ${ }^{23}$ ), se trouvant logiquement dans les conditions de pouvoir réclamer un certain droit d'initiative, voire de paternité 24. A l'initiative du coordinateur allemand du Comité de l'article 36, ce texte prévoit que chaque Etat membre devra désigner un ou plusieurs magistrats, procureurs ou officiers de police qui constitueront l'unité Eurojust et qui seront nommés «fonctionnaires de liaison ». Il s'agit ainsi en quelque sorte de regroupement des magistrats de liaison dans un simple but d'informer sur l'état des procédures et de contribuer à la coordination des enquêtes, mais sans structure propre, puisque c'est le Secrétariat général du Conseil qui est prévu pour assurer les moyens matériels ainsi que les ressources humaines d'Eurojust (interprètes, traducteurs, personnel auxiliaire, etc.). Si la filiation avec les réflexions de Schomburg est claire (partisan d'un simple centre de documentation), l'initiative vise surtout à imposer sa propre définition à un texte initial et à définir un cadre ad minima à la discussion. La réaction du Secrétariat général et des quatre présidences est vive: une réunion a même lieu à la Représentation permanente française pour convaincre le représentant allemand de retirer la proposition. Mais, en définitive, il reste sur la table du Conseil deux initiatives divergentes. Le projet des quatre présidences apparaît déjà lui-même comme un compromis : il s'agit ici d'un premier compromis à quatre, avant celui à quinze. En effet, les membres belges souhaitaient, dans la prénégociation, un instrument plus ambitieux, pour éviter avant tout le modèle dual d'Europol. Ainsi, selon Daniel Flore : «Et clairement, l'option belge de l'époque, c'était: il doit s'agir de magistrats européens; il ne faut pas refaire l'erreur d'Europol: on a des officiers de liaison près d'Europol qui sont au fond le vrai lieu de l'information et une direction d'Europol avait une dualité et une tension permanente entre le pôle européen et le pôle national au sein d'Europol. Donc l'idée était que les magistrats ne soient pas des magistrats nationaux mais des magistrats européens. Et là-dessus, la France et la Suède voulaient le raccrochement aux Etats. L'argument des négociations, c'était: puisque les Allemands parlent de magistrats de liaison, on ne peut pas faire tout le contraire. Et on est arrivé à cette notion de membre national : qui est à la fois membre d'Eurojust et national. Dans toute la décision, on voit que par certains aspects, il est vraiment un magistrat européen, mais que par l'essentiel des aspects, c'est un magistrat national qui peut en référer à son Etat. Dès la discussion entre les quatre présidences, il y a eu cette question-là, qui a été réglée par une espèce d'hybridation : membre national ${ }^{25} \%$.

On voit bien ici comment Europol, après avoir servi de déclencheur à la création d'Eurojust, joue maintenant comme contre-modèle institutionnel. Derrière cet argument, on trouve aussi une volonté de démonstration d'une plus grande efficacité de la coopération judiciaire par rapport à la coopération policière. La "véritable " négociation commence ainsi début octobre 2000 sur la base de deux textes philosophiquement différents. Emmanuel Barbe, ayant participé aux réflexions et à la 
proposition comme conseiller justice à la RP, prend, en vertu des règles de la présidence, la tête du groupe "Coopération judiciaire pénale ».

Deux dynamiques principales sont ici à observer. Sur le fond tout d'abord, l'enjeu est naturellement la base de négociations définies dans le premier pilier par la Commission qui a, dans ce cadre, comme l'a montré Pierson ${ }^{26}$, la possibilité de choisir le moment opportun pour déposer un texte. La stratégie de Barbe est alors de restreindre progressivement le choix sur un seul texte, celui des quatre présidences ${ }^{27}$. La seconde dynamique concerne la structure du groupe : il s'agit de créer en son sein une enceinte spécifique pour négocier Eurojust. On observe la volonté de créer un groupe de plus haut niveau, avec des négociateurs "moins juniors" ayant "plus de vision, des compétences plus organisationnelles que strictement juridiques». "On créait un organe, une institution" s'exclame Emmanuel Barbe. Le gestionnaire du groupe Coopération pénale, un Danois A4, technicien du droit communautaire, se trouve alors remplacé par Hans Nilsson, chef de division A3. Il semble que ce dossier soit alors considéré en quelque sorte comme l'aspect le plus "noble» de la coopération judiciaire. En témoigne d'ailleurs l'atmosphère qui se dégage des dîners ainsi organisés. Cela obéit aussi à une logique de sectorisation d'Eurojust.

La DG JAI de la Commission est passablement absente de ce jeu: plus récente, d'une composition strictement administrative et ne disposant que de peu d'expertises en matière judiciaire, elle n'a pas fait de proposition formelle avant septembre 2001. Elle s'investit par ailleurs prioritairement dans les domaines communautarisés, c'est-à-dire l'asile et l'immigration (ainsi que la coopération civile). Elle a pourtant reçu à Tampere une légitimité pour intervenir sur le pénal. Il est ainsi révélateur que le représentant de la Commission au sein du groupe de travail chargé des négociations d'Eurojust soit non pas un de ses fonctionnaires, mais un expert national détaché (END) ${ }^{28}$.

Le jeu autonome de la Commission

25 Si la Commission est véritablement absente des deux précédents jeux, c'est certes parce qu'elle n'est pas alors constituée comme acteur de la coopération judiciaire pénale, mais aussi parce qu'elle est inscrite dans un autre jeu, centré celui-ci autour de la protection des intérêts financiers communautaires et de la lutte contre la fraude. C'est dans cette perspective que, le 29 septembre 2000, prend place la proposition de la Commission de création d'un procureur européen qui va servir de véritable repoussoir et agir comme cristallisateur en faveur d'Eurojust. La proposition de Procureur est portée par la Commissaire en charge du budget, du contrôle financier et de la lutte antifraude, Michaele Schreyer, et non pas par celui en charge de la JAI, Antonio Vitorino ${ }^{29}$. Cette initiative est en effet à interpréter de façon autonome au sein d'un système relationnel assez complexe entre la Commissaire au budget, l'OLAF qui a succédé en 1999 à l'Unité de coordination antifraude (UCLAF, alors au Secrétariat général de la Commission) ${ }^{30}$, et le Parlement européen (et sa Commission du contrôle budgétaire, la Cocobu). Ce jeu comporte une forte dimension germano-allemande: l'OLAF est dirigé par Franz-Hermann Brüner, ancien procureur allemand; la Commissaire chargée du budget, Michaele Schreyer, spécialiste de politique budgétaire, appartient aux Verts allemands et a comme principal conseiller une ancienne membre du ministère de la Justice à Berlin, Margarete Hofmann; et la Cocobu est présidée par Madame Theato, de la CDU. pénale (bien que le directeur de l'OLAF soit un magistrat pénaliste d'origine), étant sous 
l'influence du Parlement européen qui vote le budget de l'OLAF. Et c'est le Parlement qui est à l'origine de l'idée de Procureur dès 1996. Le projet précis a été formulé pour la première fois en 1996 par Klaus Hansch, alors président du Parlement. L'OLAF, et en particulier son directeur "politique, législation et affaires juridiques", Claude Lecou (membre fondateur de l'UCLAF), est aussi particulièrement intéressé par la perspective du Procureur pour donner des suites autres qu'administratives à ses enquêtes, enquêtes que la Cocobu suit de façon attentive. Enfin, on assiste aussi à une juridicisation de l'OLAF, symbolisée par la mise en place, puis la montée en puissance d'une unité composée spécifiquement de magistrats. Appelée «Magistrats, conseil et suivi judiciaire ", celle-ci est directement rattachée au directeur général. L'obstacle principal de l'OLAF réside dans le fait qu'il ne dispose pas de la personnalité juridique.

Les logiques de ce jeu ont des origines anciennes : elles remontent au traité Eurocrim, à la convention "protection des intérêts financiers » de juillet 1995 et, surtout, aux premières études des années 1990 qui aboutirent au Corpus Juris de 1997, puis au Corpus Juris 2 en 2000. Mais cette proposition de Procureur par la Commission intervient alors dans une conjoncture bien spécifique, celle de la conférence intergouvernementale chargée de la réforme des traités.

CIG : unification des jeux et durcissement des clivages

Parce que la proposition de la Commission prend corps au moment de la CIG, elle a pour effet de durcir considérablement l'opposition entre les deux projets. Dans cette concurrence, Eurojust apparaît comme la seule initiative sérieuse de coopération judiciaire. Le projet de Procureur de la Commission sert alors d'opportunité à la présidence française, maîtresse des négociations jusqu'à Nice, pour proposer Eurojust comme réponse structurelle à la Commission dans le cadre de la CIG et ainsi l'inscrire dans le nouveau traité. Ce jeu de nature spécifique ne rassemble que certains acteurs nationaux de haut niveau (conseillers des ministres de la Justice mais aussi entourages des chefs d'Etat et de gouvernement), et pour la première et unique fois, le Secrétariat général du Conseil en est totalement absent.

Un acteur semble ici avoir joué un rôle décisif : il s'agit du conseiller pour les affaires internationales des ministres français de la Justice (Elisabeth Guigou puis Marylise Lebranchu) en la personne de Michel Debacq. Ayant inauguré en mars 1993 le poste de magistrat de liaison français à Rome, dont il tirera un article ${ }^{31}$, il dispose aussi de réseaux socialistes ${ }^{32}$. C'est donc par son intermédiaire que le cabinet Guigou puis Lebranchu (suite au remaniement du gouvernement Jospin en pleine présidence), s'appuyant sur le Service des affaires européennes et internationales (SAEI) du ministère, alors dirigé par Olivier de Baynast, prend une initiative importante : il rédige des propositions d'articles (30 et 31) qui consacrent juridiquement Eurojust dans le traité. L'argument de la présidence française est d'en faire mention dans le traité afin de mieux équilibrer les coopérations judiciaire et policière. Cet équilibre est assuré «topographiquement » dans le traité avec l'insertion d'un nouveau point 2 (Eurojust) : l'article 31 consacré à la coopération judiciaire comme pendant du point 2 de l'article 30 (Europol) consacré à la coopération policière.

Alors que cette question ne figurait pas dans le mandat de la CIG, il faut comprendre cette initiative comme une tentative de répondre à la Commission pour rejeter le projet de Procureur. C'est en ces termes « anti-procureur » que la position française est alors comprise. Pour cette initiative, le Secrétariat général du conseil est écarté : "On a simplement entendu après que la France avait proposé cela dans la CIG et que cela avait 
abouti ", affirme Hans Nilsson, jugeant par ailleurs l'initiative excellente. Gilles de Kerchove, quant à lui, regrette de ne pas avoir été associé à sa rédaction. Il juge que le texte proposé fait référence à des concepts dépassés, comme celui de l'extradition, « et porte encore la marque de l'ancienne approche de l'Europe judiciaire ». Mais il qualifie lui aussi l'initiative d'excellente. On voit bien ici la nature spécifique de ce jeu de la CIG qui, du fait de l'élévation de niveau dans la prise de décision, écarte momentanément certains acteurs bruxellois.

31 C'est du côté de la Commission que l'initiative française a provoqué une vive inquiétude, la référence explicite à la protection des intérêts financiers communautaires (PIF) retenant son attention. «Une mention de cette matière dans le chapitre d'Eurojust à l'époque, ça parait dangereux. à tout le moins, ça paraît brouiller les cartes et les esprits, introduire de la complexité. Au pire, ça apparaît comme un retour sur un acquis communautaire. Donc, à ce moment-là, il y a une mobilisation à la Commission", résume un membre de la Direction politique de l'OLAF (la partie de l'office intégrée à la Commission). La mobilisation de la Commission a consisté en une démarche de Madame Schreyer auprès de ses collègues qui suivent la CIG, et par une intervention de Mireille Delmas-Marty, en tant que présidente du Comité de surveillance de l'OLAF, qui écrit à ce sujet au Premier ministre et au président de la République française pour leur exposer la préoccupation de son comité. Cette mention n'apparaîtra pas dans le traité lui-même, mais seulement dans la décision Eurojust.

Cette opposition entre d'un côté, présidence française-Etats membres et, de l'autre, OLAF-Commission se durcit alors considérablement. Des rumeurs circulent, faisant de l'OLAF un vivier potentiel de recrutement pour le Procureur européen ou même, lui attribuant l'ambition de vouloir se transformer en Procureur européen. Du côté de l'office, on se défend en affirmant qu'il «se perçoit comme un service d'enquêtes, éventuellement pouvant évoluer vers un service d'enquêtes judiciaires ou être contrôlé par un organisme judiciaire communautaire, mais pas comme devenant lui-même ce procureur ou ce parquet ${ }^{33} »$. Ce dernier met par ailleurs en avant la philosophie différente des deux projets :

33 «La Commission propose des pouvoirs très intégrés, quelque chose d'ambitieux au plan de l'intégration communautaire mais on commence sur un champ de compétences matérielles étroit. Du côté de la construction Eurojust, c'est plutôt l'inverse : un champ de compétences tout de suite très large mais une montée en régime lente pour ce qui est des compétences au niveau européen. Ce sont en effet deux approches, horizontale et verticale, qui se croisent ${ }^{34}$ ».

34 Ce clivage est enfin particulièrement exacerbé par la presse. Un article des Echos de fin février 2001 prend ainsi parti en faveur du Procureur (assimilé ici à un Parquet), présenté comme un projet modèle contre Eurojust auquel est attribué tous les défauts :

" Au moment où le procès Dumas-Elf-Sirven et l'Angolagate défraient la chronique avec leurs prolongements en Allemagne ou sur les comptes des banques suisses, force est de constater que l'Europe ne se résout pas à unifier son espace judiciaire. Elle préfère la prestidigitation! A Nice, en décembre, on attendait le parquet européen, et c'est Eurojust qui est sorti du chapeau des chefs d'Etat et de gouvernement des Quinze. La France a convaincu ses partenaires de le mettre en place dès le 1er mars. Pourtant, aux yeux d'une majorité de députés européens et des fonctionnaires de la Commission qui avaient déposé sur le tapis vert de la Conférence intergouvernementale (CIG) un projet de parquet européen assorti d'un début d'harmonisation du droit pénal européen (le 
"Corpus juris"), Eurojust est un leurre... Car s'il fallait modifier les traités pour créer le parquet européen, il n'en était nul besoin pour Eurojust, simple cénacle de magistrats des chancel-leries des quinze pays de l'Union. Et pourtant, il a été inclus dans le traité de Nice, comme s'il s'était glissé dans le lit préparé pour le parquet européen... ${ }^{35}$ "

Si cet article témoigne clairement de la capacité de la Commission (et des cabinets de commissaires) à mobiliser les journalistes, il est fortement illustratif du durcissement des clivages imposé par la CIG. Mais il faut se garder de considérer la Commission comme un ensemble homogène. Celle-ci est traversée d'oppositions internes. En l'occurrence, les clivages se situent ici entre le cabinet Schreyer, la DG budget et l'OLAF d'un côté, et de l'autre la DG JAI avec, à sa tête, le commissaire Vitorino. Ce second pôle est beaucoup plus ouvert à Eurojust et même parfois critique sur le projet de Procureur. C'est ainsi que la communication de la Commission sur Eurojust datée du 22 novembre 2000 , rédigée par la DG JAI, est assez mesurée ${ }^{36}$. "Sans exclure la possibilité de présenter une proposition à ce sujet, la Commission a préféré prendre position dans le cadre d'une communication ${ }^{37}$ ", peut-on y lire en introduction. Cette démarche est inhabituelle reconnaît son auteur, Martin Wasmeier, représentant de la Commission dans le groupe de travail. La Commission propose, certes, un certain partage des tâches avec l'OLAF, mais elle affirme dans le même temps la nécessité d'un projet ambitieux : «La Commission est convaincue qu'Eurojust devrait aller bien plus loin que le réseau judiciaire européen, en marquant une étape qualitativement supérieure ». Ses recommandations concernant la forme d'Eurojust sont du même ordre : « Pour garantir une certaine indépendance, Eurojust devra avoir la personnalité juridique et disposer d'un budget propre ». Elle demande enfin la présence d'un délégué. Seule cette dernière demande, une revendication institutionnelle somme toute classique, ne sera pas acceptée en raison même du consensus des Etats membres visant à exclure la Commission de tout travail judiciaire opérationnel. La position de la DG JAI a été ensuite principalement de faire en sorte qu'Eurojust entre dans les schémas communautaires pour tout ce qui concerne la forme institutionnelle et ne soit pas, par conséquent, dérogatoire par rapport aux autres agences (comme Europol).

On retrouve par ailleurs ces mêmes clivages au Parlement européen et ses deux commissions compétentes : la Cocobu, déjà évoquée, et la Commission des libertés et des droits des citoyens, de la justice et des affaires intérieures. Si la première, comme défenseur du Procureur, s'est montrée très réservée sur Eurojust, cela n'a pas été le cas de la seconde. Et c'est elle qui donna la position du Parlement. Un groupe de parlementaires fut chargé, sous la direction de la députée allemande Evelyne Gebhardt, rapporteur du projet, de produire un rapport sur la proposition du Conseil de création d'Eurojust. Il n'y a pas lieu de s'étonner d'un accueil favorable de cette Commission parlementaire. On peut lire ainsi dans son rapport du 27 avril 2001: «Le développement de la coopération judiciaire en matière pénale face aux compétences d'Europol est une exigence de l'Etat de droit. Eurojust doit être conçu de façon à devenir l'embryon d'un futur ministère public européen ». La seule recommandation du Parlement concerne la place des policiers comme membres nationaux d'Eurojust. Il souhaite réserver cette possibilité aux seuls Etats dans lesquels les tâches des procureurs sont assumées par la police.

38 Le Parlement a joué son rôle le plus important par le soutien d'une autre de ses composantes au projet de Procureur qui a servi de contre-projet pour la présidence française dans le cadre de la CIG. Car, au total, on ne peut comprendre que le paradoxe 
qu'Eurojust, n'existant pas encore, soit déjà inscrit dans le traité, que par la spécificité du jeu de la CIG. Le coup isolé de la présidence française au sein de l'espace gouvernemental français et qui a ici provoqué des effets structurels dans un autre jeu, celui de la Commission, n'aurait pas été possible dans une autre configuration.

Clore la négociation : le jeu inversé du Conseil

Le dernier jeu a pour espace le Conseil et ses structures : groupe de travail, comité de l'article 36 (le CATS) et le Conseil des ministres JAI. Ce jeu, comme résultante en quelque sorte des quatre précédents, est celui de la décision dans un agenda saturé par le 11 septembre : elle date en effet du 6 décembre 2001. Ici la structure de décision est inversée, puisque ce sont des fonctionnaires au sein d'un groupe qui se trouvent devoir clore une négociation, alors qu'en général ce sont eux qui l'initient.

Ce jeu se présente sous deux formes : dans un premier temps les négociations de l'Unité provisoire baptisée "pro-Eurojust» (achevées en décembre 2000) et, dans un second temps, les négociations sur la décision finale qui intervient le 6 décembre 2001. Une autre particularité des négociations d'Eurojust réside dans le fait qu'une partie des premiers débats a porté en réalité sur une expérimentation et la mise en place d'une Unité provisoire préfigurant l'institution finale. Là encore, l'origine de cette idée revient au Secrétariat général du conseil. Voici le récit qu'on nous a fait au secrétariat:

41 « On s'est dit que ça allait prendre trop de temps de créer Eurojust : on va attendre cinq ans comme pour Europol. On était sous présidence française. Si on faisait une unité provisoire en attendant que le texte créant Eurojust soit tout à fait négocié, adopté... En un après-midi, on a écrit un projet très léger de création d'une unité et on l'a proposé à la ministre de la Justice, Elisabeth Guigou ${ }^{38}$ ».

42 Sans vouloir trancher l'éternelle question de l'origine des idées, c'est de nouveau le leadership du SGC, à travers son rôle de facilitateur et de conseiller du Prince qui intervient, cette fois sous la présidence française. Là encore, il s'agit bien d'un échange de bons procédés et non pas d'une imposition, impossible institutionnellement.

L'argument de mimétisme institutionnel - suivre le modèle d'Europol qui avait ouvert, dès 1994, une unité antidrogue avant sa mise en place définitive en 1999 - fonctionne bien ici. Cela correspond aussi à une contrainte des présidences qui souhaitent mettre à leur acquis durant leur court temps d'existence (six mois, en fait cinq si on exclut le mois d'août) des projets significatifs, et à une volonté propre de la présidence française de bénéficier de l'élan de Tampere. Cette "recette » institutionnelle permet aussi de proposer une réponse astucieuse, ainsi qu'une porte de sortie à la proposition concurrente de l'Allemagne. L'argument mobilisé est ici : "Comme votre projet est "light", ce sera pour un Pro-Eurojust; le vrai Eurojust, ce sera après ». Mais l'idée centrale est bien de conforter Eurojust et de le rendre inéluctable ainsi que d'orienter les négociations sur l'Unité définitive: «La présence à Bruxelles de quinze magistrats de terrain nous a été précieuse pour dessiner les contours d'une institution correspondant aux besoins réels des praticiens de l'entraide judiciaire pénale », précise de Kerchove.

En tout cas, ce projet n'étant pas considéré comme risqué par les Etats membres, les négociations avancent très vite. Lors d'une réunion informelle tenue à Marseille à la fin de juillet 2000, les ministres de la Justice donnent leur aval politique à Pro-Eurojust. Les négociations se déroulent ensuite dans le cadre du Comité à haut niveau de l'article 36 (ne rassemblant pas que des hauts fonctionnaires de la Justice, mais aussi de l'Intérieur). La décision est adoptée par le Conseil JAI du 14 décembre 2000 quelques 
jours après le sommet de Nice. Elle est directement applicable à tous les Etats membres. La seule opposition est venue de la Commission (DG JAI), qui a estimé qu'il n'y avait pas d'urgence, et que cette solution transitoire ne devait pas durer. Cette position a été relayée par le rapporteur du projet au Parlement.

La conséquence immédiate est l'arrivée, le 1er mars 2001, au sein du Secrétariat général $\mathrm{du}$ conseil, de quinze magistrats formant Pro-Eurojust. Jusqu'à l'adoption de l'instrument définitif, l'Unité est présidée par le représentant national de l'Etat qui exerce la présidence de l'Union européenne (M. Björn Blomqvist, suédois). La France choisit comme représentant Olivier de Baynast, jusque-là le chef du SAEI. Le climat est enthousiaste. Hans Nilsson organise ce soir-là un dîner à son domicile: "Il y avait beaucoup de gens et c'était vraiment presque euphorique. C'était: "Maintenant, nous avons créé quelque chose" " se souvient-il. On retrouve ici encore cette spécificité de création institutionnelle, mais également une transformation des modalités du travail judiciaire. En effet, beaucoup des membres de Pro-Eurojust arrivant à Bruxelles avaient rencontré des difficultés dans l'exercice de leur fonction et pouvaient alors se retrouver dans une enceinte commune.

Alors que l'Unité provisoire est mise en place, il reste enfin à négocier la décision finale. Il reste deux présidences suivant l'échéancier de Tampere : la présidence suédoise et la présidence belge. La négociation reprend au sein du groupe de travail du Conseil (coopération judiciaire pénale), réuni en session restreinte Eurojust, comme on l'a déjà $\mathrm{vu}$, avec Emmanuel Barbe. La montée en puissance se manifeste avec la nomination, par la Suède, d'un président de groupe pour Eurojust, en la personne de Peter Stromberg. Si la présidence française a servi essentiellement à faire le tour du texte, à identifier les points à discuter, il n'y avait, au moment de Nice, un consensus que sur les missions générales, les compétences et la structure (membre national et collège). Les négociations commencent alors avec la présidence suédoise sur des questions plus techniques, principalement la protection des données et les structures (comme l'audit et le directeur administratif).

C'est à la présidence belge qu'il revient de clore la négociation. Ici le processus d'élévation du profil du président du groupe Eurojust se poursuit : Daniel Flore, qui a suivi toutes les négociations, préside désormais à la fois le groupe Eurojust et le Comité à haut niveau de l'article 36 (CATS), suite à un changement interne au ministère belge de la Justice. Il entend se réaffirmer par rapport au SGC. Pour arriver à une décision d'ici la fin de sa présidence, il prévoit pas moins de treize jours de réunion, c'est-à-dire plus que l'ensemble des trois précédentes présidences pour toute la coopération pénale. Il joue aussi de sa position de président du CATS pour mettre les membres du groupe devant leurs responsabilités : "Je suis parti de l'idée que Tampere engageait tous les gouvernements et que donc ce n'était pas à un fonctionnaire dans un groupe de travail de contrecarrer la volonté politique d'aboutir en décembre $2001{ }^{39}$ ». Hans Nilsson, toujours organisateur du groupe, se souvient lui de n'avoir «jamais travaillé moins de 80 heures par semaine sous la présidence belge ». Il ajoute que "c'était la seule fois où je me suis demandé s'il ne fallait pas m'installer un lit ici ${ }^{40}$ ».

48 Dans cette configuration, le 11 septembre rend certaines querelles sur Eurojust assez dérisoires, d'autant que l'agenda est vite surchargé par d'autres projets: le mandat d'arrêt européen et la définition du terrorisme à l'ordre du jour du Conseil exceptionnel du 20 septembre, puis du Conseil européen spécial du 21 septembre. Ces deux derniers projets, ainsi qu'Eurojust, se trouveront tous adoptés au Conseil JAI du 6 
décembre 2001. Le 11 décembre 2001, ironie du sort, hasard de calendrier ou volonté de revanche, la Commission publie son Livre vert sur le Procureur européen ${ }^{41}$.

La décision officielle de création d'Eurojust intervient le 28 février 2002 et est publiée au Jo le 6 mars, date de son entrée en vigueur. Hébergé comme Pro-Eurojust au Secrétariat général du Conseil, Eurojust s'installe le 10 décembre 2002 à La Haye. L'inauguration officielle eut lieu le 29 avril 2003. Le choix de La Haye - en raison de la présence d'Europol ${ }^{42}$ - entériné au Conseil européen de Laeken en décembre 2001, est définitivement confirmé par une décision du 13 décembre $2003{ }^{43}$. Le 14 avril 2003, Eurojust signe enfin un protocole d'accord avec l'OLAF, réglant la question des modalités de leur coopération puis fait de même avec Europol le 9 juin 2004.

Le Livre vert sur le Procureur de la Commission est à étudier dans le cadre d'un dernier jeu institutionnel, celui de la Convention, puis d'une nouvelle CIG qui a donné naissance à la Constitution. C'est en effet dans ces deux enceintes qu'une seconde mise en concurrence eut lieu entre le projet de Procureur et Eurojust. Cette nouvelle négociation tourna à l'avantage d'Eurojust. Le compromis trouvé par le præsidium a été de maintenir la possibilité de créer le Procureur à la tête d'un Parquet mais «à partir d'Eurojust » et à l'unanimité, ce qui ruine ses chances. C'est cette même formulation qui a été reprise en CIG et donc inscrite dans la Constitution adoptée le 18 juin 2004 par le Conseil européen de Bruxelles et signée à Rome le 29 octobre 2004 (article III-274). Ce texte consacre parallèlement Eurojust dans son article précédent.

51 La décision de création d'Eurojust peut ainsi s'expliquer par une série de cinq jeux institutionnels, six si l'on compte celui de la Convention-Constitution. Ces jeux ont fait agir une pluralité d'acteurs et c'est ainsi que ce projet, aux origines aussi anciennes qu'Europol, peut avoir plusieurs paternités: allemande, française ou belge selon l'implication de ces Etats dans tel ou tel jeu. Une autre paternité a même été ensuite invoquée, celle de l'Italie qui met en avant le modèle institutionnel de sa Direction générale anti-mafia. S'il y a une paternité à rechercher, il s'agit de celle du Secrétariat général du Conseil, mais celle-ci est impossible à revendiquer et se voit donc niée officiellement par beaucoup des autres protagonistes. Le rôle de certains magistrats nationaux, désireux de faire de la coopération institutionnelle, a aussi été déterminant. Eurojust a commencé son action dès 2002 (2001 pour Pro-Eurojust) à Bruxelles puis à La Haye (siégeant dans le même bâtiment que la Cour pénale internationale). Il serait utile dans ce cadre de poursuivre l'analyse en observant la mise en place de cet organe et les usages de cette création institutionnelle par les magistrats nationaux, usages qui pourraient conduire à certaines formes de renationalisation.

Mais ce qui est le plus frappant est la discussion permanente sur sa structure institutionnelle. Ainsi, le projet de texte constitutionnel prévoit de renvoyer à une future loi la définition définitive d'Eurojust. Et le Conseil européen des 4 et 5 novembre 2004 qui a lancé le programme pluriannuel dit « Programme de La Haye » a fixé au 1er janvier 2008 au plus tard l'adoption d'une telle loi, sur proposition de la Commission. Aussi, c'est bien à une renégociation à laquelle nous allons assister, faisant appel de nouveau à cette spécialité européenne qu'est l'innovation institutionnelle ${ }^{44}$. 


\section{NOTES}

1.. Action commune du 22 avril 1996 (JOCE, 27 avril 1996).

2.. Action commune du 29 juin 1998 (JOCE, 7 juillet 1998).

3.. Ancien juge suédois, Hans Nilsson, alors en poste à Strasbourg au Conseil de l'Europe au sein de la division des problèmes criminels de la direction des Affaires juridiques, évoque une discussion dès 1991, dans un voyage en train en compagnie de Wolfgang Schomburg, ancien procureur et alors secrétaire d'Etat à la Justice au Land de Berlin et d'un professeur allemand (entretien, juillet 2003).

4.. Nous avons procédé à une douzaine d'entretiens avec des (les) acteurs qui ont participé à la naissance d'Eurojust, repérés selon la méthode de la «boule de neige ». Cette étude s'insère dans un contrat de recherche achevé en mai 2004 avec Hélène Michel et Natacha Paris pour la Mission de recherche Droit et Justice (Mobilisations contre la criminalité organisée et institutionnalisation d'un espace judiciaire pénal européen 1996-2001).

5.. Selznick P., Leadership in Administration. A Sociological Interpretation, New York, Peterson, 1957.

6.. Jamous $\mathrm{H}$., Sociologie de la décision : la réforme des études médicales et des structures hospitalières, Paris, Editions du CNRS, 1969.

7.. Friedberg E., Le Pouvoir et la Règle. Dynamiques de l'action organisée, Paris, Seuil, 1997, (1re édition 1993), p. 160.

8.. Allison G., Essence of Decision. Explaining The Cuban Missile Crisis, Boston, Little Brown, 1971.

9.. Organisation que nous avons étudiée par ailleurs, voir Mangenot M., « Une Chancellerie du Prince. Le Secrétariat général du conseil dans le processus de décision bruxellois ", Politique européenne, $n^{\circ} 11$, automne 2003.

10.. Le Groupe de TREVI (Terrorisme, Radicalisme, Extrémisme, Violence Internationale), fondé à Rome le 1er décembre 1975, est la première initiative européenne dans le domaine de la coopération policière et judiciaire mais strictement intergouvernementale. D'abord centrée autour de la lutte contre le terrorisme, cette coopération s'est élargie avec TREVI II puis III.

11.. Il a été remplacé le 15 mars 2003 par un autre Britannique issu, lui, du cabinet de Leon Brittan et devenu directeur général adjoint de la Concurrence puis directeur général de la Presse et de la Communication.

12.. Il en sera de même ensuite quand, en 1999, une directrice de la Coopération judiciaire et policière sera nommée en la personne de Denise Sorasio, fonctionnaire du service juridique de la Commission mais surtout chef de cabinet du président du Parlement européen.

13.. Entretien avec Hans Nilsson, juillet 2003.

14.. Il faut signaler aussi le choc culturel qu'a représenté pour les ministères de l'Intérieur l'intégration de la coopération «TREVI » au sein de l'Union européenne et la soumission du processus décisionnel aux règles et procédures du Conseil. Comme le fait remarquer Gilles de Kerchove : « les policiers sont, par nature, plus tournés vers l'action opérationnelle que la délibération législative. Comme le Conseil des ministres est essentiellement une institution qui produit de la norme, les policiers y sont moins à l'aise que leurs collègues des ministères de la Justice, dont c'est la fonction première. 
Un des défis à relever consiste à aménager des procédures et imaginer des méthodes permettant aux responsables des polices européennes de coordonner des opérations de police à l'échelle de l'Union et de participer à la définition d'une politique criminelle européenne », entretien, juillet 2002.

15.. Note de la présidence au COREPER/Conseil, Bruxelles, 22 novembre 1996.

16.. A l'université libre de Bruxelles, à l'université catholique de Louvain et aux facultés universitaires Saint-Louis pour de Kerchove, au Collège d'Europe à Bruges pour Nilsson et à l'université catholique de Louvain pour Daniel Flore, également ancien assistant. 17.. Diplomate allemand, il était pourtant sur le départ puisque qu'il a été remplacé, juste après le Conseil de Tampere, le 18 octobre 1999, par le duo Javier Solana comme Secrétaire général et haut représentant pour la PESC et Pierre de Boissieu, comme Secrétaire général adjoint.

18.. Il s'agit de Wolfgang Schomburg.

19.. Entretien, juillet 2002.

20.. Organe chargé de la coordination de la coopération judiciaire et policière, qui fait suite au K4.

21.. Document du Conseil 10356/00 EUROJUST 7 et 10357/00 EUROJUST 8. Il faut noter que le rédacteur d'origine de ce texte est le Secrétariat général du conseil.

22.. Initiative de la République portugaise, de la République française, du royaume de Suède et du royaume de Belgique en vue de l'adoption de la décision du Conseil instituant Eurojust afin de renforcer la lutte contre les formes graves de criminalité organisée [Journal officiel C 243 du 24 août 2000].

23.. Initiative de la République fédérale d'Allemagne en vue de l'adoption d'une décision du Conseil relative à la création d'une unité « Eurojust » [Journal officiel C 206 du 19.07.2000].

24.. En raison du rôle antérieur joué par Wolfgang Schomburg dans le « lancement » de l'idée en 1991 avec Hans Nilsson puis surtout auprès de sa ministre à Turku en septembre 1999.

25.. Entretien, mai 2003. Voir aussi son article : Flore D., « D'un réseau judiciaire européen à une juridiction pénale européenne : Eurojust et l'émergence d'un système de justice pénale », de Kerchove G., Weyemberg A. (dir.), L’Espace pénal européen : enjeux et perspectives, Bruxelles, Presses de l'université de Bruxelles, 2002. Il y précise par exemple p. 16 que les officiers de liaison sont des « corps étrangers » à l'organe européen alors que la direction d'Europol, c'est-à-dire le directeur, les directeurs adjoints et le personnel, est garante de l'intérêt européen.

26.. Pierson P., « The Path to European Integration. A Historical Institutionalist Analysis », Comparative Political Studies, vol. 29, n², avril 1996.

27.. Lors de sa réunion du 5 septembre 2000, le Comité de l'article 36 avait demandé au groupe de travail de procéder à l'analyse des deux textes et de les combiner en un seul. 28.. Martin Wasmeier, procureur allemand (ayant depuis intégré les cadres de la Commission).

29.. On peut noter que ces deux portefeuilles étaient auparavant réunis dans les mains d'une seule personne : la Commissaire suédoise, Anita Gradin, qui n'eut qu'une très faible autorité.

30.. Créé sous le nom d'UCLAF, l'OLAF a reçu le 1er juin 1999 un statut d'indépendance pour effectuer les enquêtes antifraude internes (tous les organes et institutions de l'Union européenne) et externes (tous les destinataires ou les débiteurs des fonds communautaires). 
31.. Debacq M., « Politiques nationales et dimension européenne de la lutte contre la criminalité organisée », Relations internationales et stratégiques, n²0, hiver 1995. 32. Nommé en mai 2001 à la tête de la section antiterroriste du Parquet de Paris, il a été démis de ses fonctions le 1er mars 2004, suite à l'affaire AZF.

33.. Entretien avec Sébastien Combeaud, membre de la direction politique de l'OLAF, juillet 2003.

34.. Entretien, juillet 2003.

35.. Royo M., «L'Europe judiciaire à reculons », Les Echos, 21 février 2001.

36. Communication de la Commission concernant la création d'Eurojust [COM(2000)746 final, non publieé au Journal officiel], 22 novembre 2000.

37.. Entretien, juillet 2003.

38.. Entretien, mai 2003.

39.. Entretien, mai 2003.

40.. Entretien, juillet 2003.

41.. Commission européenne, Livre vert sur la création d'un Procureur européen pour assurer la protection pénale des intérêts financiers communautaires, Bruxelles, 11 décembre 2001 (COM (2001)7515 final.

42.. L'autre hypothèse semble avoir été Luxembourg.

43.. Décision des représentants des Etats membres réunis au niveau des chefs d'Etat et de gouvernement, 13 décembre 2003, JO du 3 février 2004.

44.. Cette caractéristique observée pour la PESC par Yves Buchet de Neuilly est loin ainsi d'être spécifique à ce pilier ou à la politique extérieure au sens large. Voir « Des professionnels de l'innovation institutionnelle », Georgakakis D., (dir.) Les Métiers de l'Europe politique, Strasbourg, Presses universitaires de Strasbourg, 2002.

\section{RÉSUMÉS}

Eurojust est le nouvel organe de coopération judiciaire en matière pénale de l'Union européenne. Cet article consiste en une sociologie de la décision de sa création, analysée en termes de « jeux institutionnels ». La genèse d'Eurojust est illustrative de certaines spécificités des configurations institutionnelles européennes et interactions bruxelloises entre fonctionnaires, magistrats et ministres. Elle donne à voir le leadership du Secrétariat général du Conseil, la socialisation et la spécialisation d'un groupe aux ressources intellectuelles élevées et intéressé au travail « noble » d'innovation institutionnelle. Cet article revient sur les effets déterminants d'une forte concurrence inter-institutionnelle avec la Commission et l'OLAF pris dans une logique autonome et parlementaire, sur le travail spécifique de la présidence ou des présidences et, enfin, sur le rôle décisif de la conjoncture de Conférence intergouvernementale qui, élevant le niveau de la décision, seule, permet certains coups.

Eurojust is the new judiciary cooperation organ of the European Union. This article offers a sociology of the decision that generated its creation. This decision is analysed in terms of "institutional games". The genesis of Eurojust illustrates the specificity of the European institutional configurations and of the interactions taking place in Brussels between officials, judges, and ministers. It also shows the leadership taken by the European Council's office of the 
secretary general, the socialization and the specialization of a group with high standard intellectual resources, willing to participate to the "noble" task of institutional innovation. This paper goes back over the determining effects of a strong inter-institutional competition with the Commission and OLAF, caught in a autonomous and parliamentary logic. The paper also goes back on the specific workings of the presidency or presidencies, and lastly, on the decisive role of the conjuncture of intergovernmental conferences, which, by elevating the level of decision, enables specific coups.

\section{INDEX}

Index géographique : Union européenne

Mots-clés : bureaucraties/ théorie des organisations, construction européenne, institutions politiques et agences, juge

Thèmes : EUROJUST

\section{AUTEUR}

\section{MICHEL MANGENOT}

Michel Mangenot est Maître de conférences à l'université Robert Schuman de Strasbourg, membre du Groupe de Sociologie Politique Européenne - PRISME (CNRS UMR 7012). 